\title{
Intelligent Manufacturing Production Line Simulation of Super Capacitor
}

\author{
$\mathrm{Li} \mathrm{He}{ }^{1}$, Tengyu $\mathrm{Li}^{2}$, Bin $\mathrm{He}^{3 *}$ \\ ${ }^{1,2,3}$ School of Mechatronic Engineering and Automation, Shanghai University, China \\ Email: lihe.shu@foxmail.com, islitengyu@foxmail.com,mehebin@gmail.com
}

*Corresponding Author

\begin{abstract}
The production line of a super capacitor is a complex production line, and so many factors need to be considered during the designing process. In this paper, the plant simulation software of Siemens company is used to simulate the super capacitor production line, to verify the rationality of the application of digital twin technology in the complex production line designing process. Using the approximate parameters to study the influence of Automated Guided Vehicle (AGV) characteristics on the production line. Simulation in software environment can well demonstrate the actual production process. It is convenient to model the production line in the software environment. The malfunction and other factors in the actual production process can be replaced in software as the failure rate in the simulation environment. Data statistics and other functions can directly see the changes in various parameters in the production line, to get a better optimization scheme. The changes in actual can be "copied" to the simulating process, which will make benefit in establishing a production line.
\end{abstract}

\section{Keywords-Digital twins, production lines}

\section{INTRODUCTION}

With the development of sensing technology, the improving of software and hardware technology and computer operation ability, the digital twin technology has been further developed with the promotion of industrial intelligence and industrial information system. Digital twin technology was gradually applied to the simulation design and optimization of a complex production line.

Super capacitor is a new type of energy storage device, which can reach farad capacity in a tiny volume. There is no need for special charging circuit and control discharge circuit since overcharge and over-discharge have no negative impact on its life compared with battery. From the perspective of environmental protection, it is a green energy. Super capacitor can be welded. Therefore, there are no problems such as weak battery contact. The application prospect of super capacitors in new energy transportation vehicles is exceptionally broad.

In the design and application of complex production line, there are many processing procedures during the production process, and in the optimization process, the modification of a single process often leads to changes in other processes, which makes the design and application optimization more difficult. Therefore, an efficient and convenient design method is urgently needed.

With the development of digital twin technology, the use of plant production line simulation software for digital twin simulation can make 2D drawings into a 3D model environment for optimal design. Many researchers have done many researches on this kind of problems by using simulation software. Some researchers optimized the existing projects with Flexsim software. $\mathrm{Wu}, \mathrm{Xu}$ et al established new models to promotes the understanding of structural optimization methods and the application of simulation models [1]. Wan, Gao and $\mathrm{Xu}$ built a model of actual production line then simulated to find the problems [2]. Sun used Flexsim software to model the original system then proposed a new classification to optimize the system [3]. Jiang, Ma and Liu simulated the actual situation by manual simulation and computer simulation. Finally, use the computer to evaluate two simulations [4]. Rao, Nallusamy and Raman aimed to enhance productivity by simulating using Flexsim software [5]. The research of Pongjetanapong and O'Sullivan et al applied the HCCM framework to a real-world Cytology lab (with complex decision making for task allocations) and demonstrated how the resultant conceptual model could be implemented within an off-the-shelf healthcare simulation package (Flexsim HC) [6]. Peng simulated the running of the original program to find out the bottlenecks in the production then changed the various parameters of the production logistics to get the improved program[7]. Wang and Nie built a 3D simulation platform to simulate different operation scheduling modes of rail-mounted gantry crane in railway container central station then simulated different loadingunloading modes and compared them in Flexsim software [8]. Satish Kumar and Sanjeev et al think that to schedule the dynamic environment of a Flexible manufacturing system (FSM), discrete event simulation is the right choice. They used Flexsim finish their study [9]. Satish Kumar and Janardhana Raju deals with a simulation study to investigate how to optimize manufacturing time in Flexible Manufacturing Systems by Flexsim software [10]. Su and Ma et al took the warehousing operation system of a logistics center as an example to study the application of Flexsim simulation in the warehouse operation process [11]. He and Hu established a model of the enterprise operating system and to measure the service capabilities of the enterprise through the simulation of the model [12]. In paper of Chen, Hu and $\mathrm{Xu}$, based on a comprehensive analysis of the simulation characteristics of the highway freight terminal and Flexsim simulation methods, a typical road freight station was used for an example to establish the simulation models and gave a simulation result of the area ratio of the different technical levels of highway freight terminal [13]. Zhang and Tian 
Zhang and Tian simulated, analyzed and evaluated the design scheme through the establishment of the Flexsim simulation model [14]. Ge and Zhu et al proposed the idea of establishing a semi-automatic sorting system. They simulate the express sorting process in Flexsim software [15]. Chang and Xu et al used the Flexsim software to simulate the improved assembly process and verify the feasibility of the scheme [16]. In paper of $\mathrm{Ge}$ and $\mathrm{Li}$ et al, the simulation based on Flexsim was applied to find out the bottleneck station and unreasonable factors. The improvement is carried out from layout, site management and motion. The proposed program was verified by simulation [17]. Huang and Xu used the Flexsim system simulation software to simulate outbound workflows of warehouse plan to obtain a better design scheme [18]. Tian and $\mathrm{Ze}$ et al combined with the blocking phenomenon in the simulation and the data analysis after the simulation, the "bottleneck" problem in the wheelset pressing line is determined [19]. In article of Riskadayanti and Yuniaristanto, Flexsim software was used to model the actual production system. Analysis of the results of the simulation conducted to determine the utility level of each machine [20]. Zhang and Duan et al combined Flexsim simulation software to simulate and optimize the sofa production process which would make the production more smooth, balanced, orderly and efficient [21]. In paper of Zhou, Yang and You, the experience ranking method was used to reasonably sort the bottleneck work station and other work stations of the packaging line, in order to balance the load for each station, and build the optimized packing line by Flexsim simulation model to verify its effectiveness [22]. Jin and Li proposed idea of using Flexsim software to improve the distribution system. It was found that the new strategy was more reasonable [23]. Wang and Xu et al used Flexsim software study the capacity of Tonggu channel in Shenzhen Port. The research results can provide important decision support basis for channel construction scheme [24]. In view of the low productivity, Bao and Liu analyzed the operation flow of the assembly line of X Motor factory, and the bottleneck station was analyzed by Flexsim simulation [25]. Liu, Lu and Wang think it is an effective way to optimize the layout of the facilities of the automated warehouse by using the Flexsim software simulation technology [26]. Jia and Cao used Flexsim software to optimize the existing assembly process of a minivan manufacturing plant [27]. Ying and Liu took the end logistics distribution in the university as the research object [28]. Base on operating data on one typical flight day, Zhou and Tang proposed a simulation model is built by Flexsim, simulating ground operation of flights under two optimization schemes [29]. Fang, Li and Zhang used Flexsim to establish the simulation model of the logistics and transportation process of the factory area at the current stage, and obtained the indicators such as use efficiency of each transport vehicle in the area and the average time of transportation for the orders [30]. Most of researchers used Flexsim software to finish their study. Plant Simulation is a simulation software of Siemens. Compared with the Flexsim software, Plant Simulation software is easier to use. Programming language is easier to understand. In this paper, Plant Simulation software is used to simulate production line of super capacitor, which is a kind of intelligent manufacturing production lines.
It will make simulations of complex production lines more convenient but also feasible.

This paper uses plant simulation software to establish the digital model of super capacitor production line, and verifies the rationality of digital twin technology in the design and application optimization process of complex production line such as super capacitor. Finally, it proves the feasibility of the simulation in Plant Simulation software.

\section{ESTABLISHMENT OF SUPERCAPACITOR PRODUCTION LINE MODEL.}

After consulting the company's production data, the production process diagram of super capacitor is shown in Figure1.

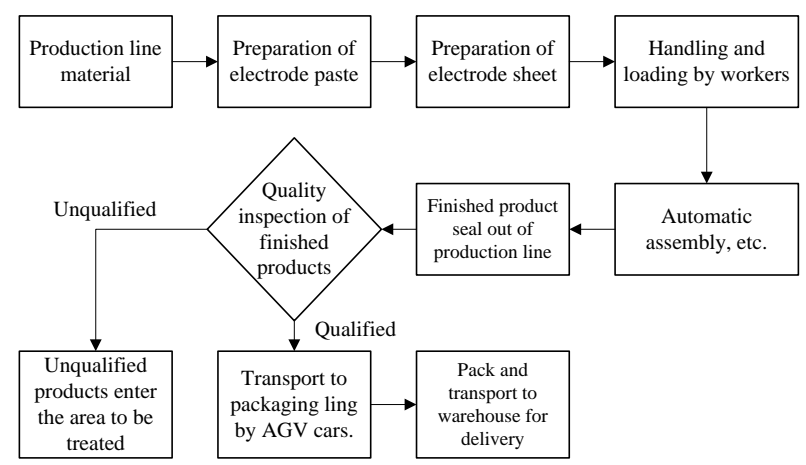

Fig 1 The production process diagram of super capacitor.

According to the flow chart and the information of the production machine, the digital model of the super capacitor production line is built in the plant simulation software to establish the model basis for the subsequent simulation.

In plant simulations, a material source is needed to be added as the material inlet of the production line. The conveyor in the software can be used as the band carrier in the actual production line. The connection between special stations is carried out by workers. For example, in the actual production line, the polar coil produced by the coater needs to be manually transported to the winding machine. In some positions, the transfer robot can be used to transport the materials.

The produced products need to be transported to the inspection line or other production lines in. In order to realize the automation of production, AGV is selected to carry out the final material handling work. The running AGV is set as a circle, which makes the AGV can carry out the transportation operation repeatedly.

At the same time, a detection area is added to the moving track of the car. Press and hold down the rail path area, and then detect the position of the rail. Enter the 3D window of the station, delete the original station model, and use the modeling tools in the software to build a cuboid model of the detection area to represent the actual detection area. Place the material source near the detection area. Find the Mu option in the pop-up dialogue box. Drag the trolley icon under the mobile unit label in the toolbox to the Mu blank space in the dialog box. Use the connector to connect the material source with the detection area.

Enter the programming environment and enter the program which means stopping the AGV from the detection 
area at the loading point, and then load the parts in the buffer zone onto the AGV for transportation. When the car is full of parts, then cars start transportation. Create a "select and place" in the appropriate position, so that the AGV can stop in the operating range of the transfer robot. When dragging to the track, the transfer robot will automatically return to its original position. Currently, select the unload part option. At this time, a sensor is automatically created. Its function is to stop the car which full of parts at this position, and the transfer robot will unload the parts on the cars. Place the material termination model in the working range of the transfer robot. Connect the transfer robot with the material termination model by a connector.

The purpose of product quality inspection is to detect inferior products for screening. Therefore, it is necessary to set the attributes of products randomly on the inspection line. In the actual production line, the quality of products cannot be distinguished by the appearance of apparent colors. To realize the simulation of the actual inspection process, a particular attribute of parts is changed. Through the method of converter additional control, the goal of distinguishing the products and bad products on the detection line is realized.

Based on the station object simulation to replace the processing machine needed by each station on the actual production line; based on Simtalk programming language to write the loading and unloading procedures of transfer robot, the color change program of products after processing in the station and the program of randomly producing unqualified products. The quality of products is screened by the converter, and the number statistics module is set at the end of the material. The complete production model is shown in Figure 2.

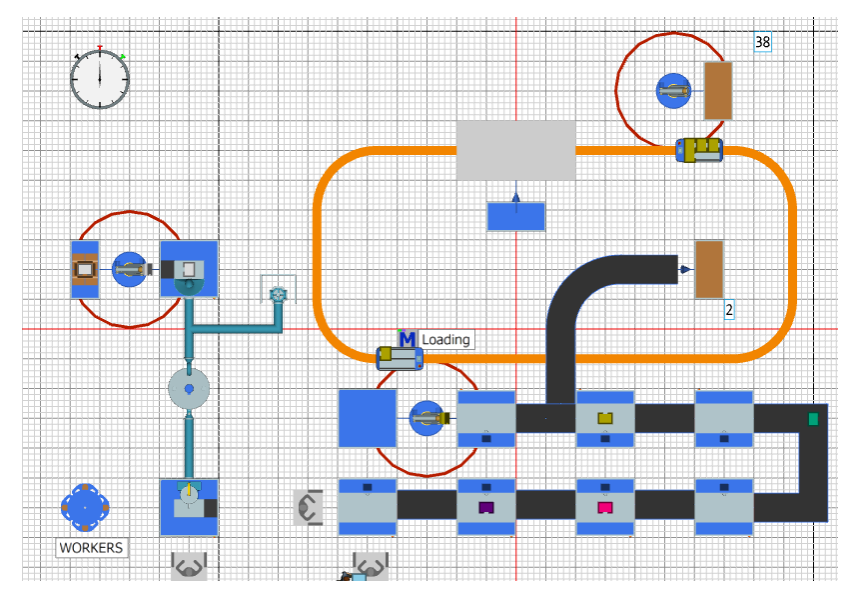

Fig 2 The digital model.

According to the actual processing time data, the processing time is set for simulation. Because the production line will stop due to the equipment failure in the actual production process, the failure rate of the processing machine is set to $5 \%$. After failure happened, the maintenance worker is set to carry out the equipment maintenance work. The preliminary statistical report is obtained after running the simulation, as shown in Table 1.

TABLE 1 THE PRELIMINARY STATISTICAL REPORT.

\begin{tabular}{|l|l|l|l|}
\hline Name & Working & Waiting & Failure \\
\hline Workstation1 & $50.12 \%$ & $45.26 \%$ & $4.62 \%$ \\
\hline Workstation2 & $74.30 \%$ & $25.70 \%$ & $0.00 \%$ \\
\hline Conveyor & $100.00 \%$ & $0.00 \%$ & $0.00 \%$ \\
\hline RobertArm1 & $51.94 \%$ & $48.06 \%$ & $0.00 \%$ \\
\hline RobertArm2 & $22.57 \%$ & $77.43 \%$ & $0.00 \%$ \\
\hline AGV Pathway & $28.30 \%$ & $71.70 \%$ & $0.00 \%$ \\
\hline
\end{tabular}

The figures in the table show the result of the simulation. As shown in the table, decrease the waiting/ time is one of the ways to optimize.

According to the actual production situation, the factors not considered in the simulation are as follows.

1. There is no simulation in the process of shift handover.

2. During the actual operation of the equipment, the transportation speed and production efficiency will fluctuate, but after a long time of simulation, the impact of the fluctuation will gradually reduce.

3. In the actual production process, there are some reasons to produce waste products.

Based on the above analysis, these reasons will not seriously affect the actual capacity of super capacitor, and the simulation results have certain accuracy.

\section{SIMULATION OF PRODUCTION LINES}

The processing time of each workstation can be set by yourself. After consulting the relevant product information, the relevant data is set up in the software. Set the processing time to check the value. In order to study the working state of the AGV and robot arm, the processing time of each station is set to the same value, so that the production line will not be blocked.

After the simulation of the production line is started, the production status of each station can be comprehensively perceived in the software by opening the dialogue box of the workstation.

Under the premise of ensuring the production line is running well and not blocked, the processing time of each station is set to 10 basic units. Based on this premise, the influence of the speed of the robot arm on the operation of the production line is studied. Taking the super capacitor assembly line as an example, the influence of the operation parameters of the $\mathrm{AGV}$ and the transfer robot on the production line is studied.

\section{A. The influence of robot arm handling speed on assembly line operation speed.}

During the producing process of the entire assembly line, the robot arm is used to move the production in the feeding position and the cutting position. First, study the transfer robot in the feeding position.

Since the next working position of the feeding port is a blender, the working mode of the mixer is to enter the liquid and fill the whole agitator volume before starting operation. Therefore, the running speed of the transfer robot will affect the production of subsequent workstations. When the mixer is blocked, the whole production line is in a state of suspension. The speed of transfer robot transportation will affect the suspension time of the production line, and the length of the transfer robot will be transported. It will reduce the duration of the whole production line. 
To observe the influence of the transfer robot on the production line, the conveying direction of the conveying robot is moved to the buffer zone, and the parts with buffer parts are automatically transferred to the car. That is to say, when the speed of the transfer robot cannot meet the requirement of timely conveying the parts to the AGV, the parts of the buffer zone will be stuck, because the processing time of each station is the same. So, the whole production line is produced at the speed of 10 s for each part.

\section{$B$. Influence of $A G V$ logistics handling speed on the production line.}

The logistics handling system of the AGV is used in the transportation process of the next station or production line when the production is finished. The operation track of the AGV is simplified to the ring type. The AGV starts to stay in the testing area for a certain period of time (controllable time). After the car reaches and loaded with parts, it stops at the unloading point until the transfer robot unloads all the parts and then goes back to the monitoring point. When one transportation cycle is completed, the time at which the AGV is loaded at the loading point and the unloading point is affected by the handling speed of the robot arm, and the time to stay on the track and the detection area is basically constant

The parameters of the material source, a fluid source, stirrer and other workstations are used to study the transfer robot. The speed of the transfer robot and the production speed of the production line are the same so as to prevent the operation speed of the transfer robot from affecting the running state of the production line. The running speed of the AGV will directly affect the operation of the production line. When the carrying capacity is constant, that is, the speed of the production parts of the production line is constant, the capacity of the AGV cart's logistics handling system will mainly depend on the speed of the AGV and the number of AGV.

After setting up the relevant parameters, the simulation of the production line is started, and the simulation speed is adjusted to the maximum value. After running for a period of time, it is found that the parts of the buffer area are stacked and stuck. This indicates that the conveying capacity of the AGV cannot meet the demand of the production line for the speed of the material handling, while the parts of the buffer zone are stuck, which means that the production continues. When the number of buffer parts reaches the maximum value of the buffer capacity, parts cannot enter the buffer zone, which will cause blockage in the production line, which will affect the regular operation of the production line. If the speed of the AGV is improved, more hidden trouble will show up in the actual production process.

After setting the speed of AGV to a constant value and increasing the number of AGV trolley running on the track, it is found that in the course of operation, the AGV cart's logistics handling system can meet the production output speed requirement of the production line. The phenomenon of waiting for AGV queue appears before the loading point of the robot arm, that is, the excess number of AGV and the increase of logistics cost in the actual production line.

In a word, the carrying capacity of the AGV cannot meet the output speed of the production line, which will further lead to the blockage of the production line and lead to the paralysis of the production line. When the carrying capacity of the AGV is far greater than the output capacity of the production line, it will lead to higher logistics handling costs.

The handling speed of the logistics handling system needs to be slightly faster than or equal to the output speed of the production line.

\section{CONCLUSION}

After the establishment of the 3D model of the supercapacitor production line, the simulation of the production line is completed in the virtual space by using the relevant production line data obtained from the investigation. At the same time, the influence of the relevant parameters of the $\mathrm{AGV}$ on the operation of the production line is studied. From 3D modeling to simulation, the application of digital twins technology in the process of production line design has been verified. It is feasible that simulating of the production line in plant simulation software.

\section{ACKNOWLEDGEMENTS}

The work was supported by the Shanghai Science and Technology Committee Project (Grant No.19511104702).

\section{REFERENCES}

[1] S. Wu, A. Xu, W. Song, and X. Li, "Structural Optimization of the Production Process in Steel Plants Based on Flexsim Simulation," Steel Research International, vol. 90, no. 10, 2019.

[2] J. Y. Wan, L. Y. Gao, and Z. F. Xu, "Research on MP-X Assembly Line Planning Simulation Based on Flexsim," Applied Mechanics and Materials, vol. 2025, 2012.

[3] X. Sun, "Simulations on the Spare Parts Inventory Management of Equipment Maintenance in Manufacturing Enterprise Based on Flexsim," Advanced Materials Research, vol. 2450, 2013.

[4] J. Rongfen, M. Xiaoxia, and L. Tongjuan, "The Simulation and Optimization Research of a Filling Station Based on Flexsim," Journal of Applied Science and Engineering Innovation, vol. 3, no. 3, 2016.

[5] G. V. P. Rao, S. Nallusamy, and P. Raman, "Enhancement of Production in Subassembly Line of a Medium Scale Industry Using Different Lean Tools and Flexsim Simulation Software," International Journal of Engineering Research in Africa, vol. 4743, 2019.

[6] K. Pongjetanapong, M. O’Sullivan, C. Walker, and N. Furian, "Implementing complex task allocation in a cytology lab via HCCM using Flexsim HC," Simulation Modelling Practice and Theory, vol. 86, 2018.

[7] J. L. Peng, "Optimization on the Improvement Schemes of Production Logistics System Based on Flexsim Simulation," Advanced Materials Research, vol. 1250, 2011.

[8] X. Nie and L. Wang, "Simulation Process Design for Scheduling Mode of Railway Container Terminals based on Flexsim," Journal of Physics: Conference Series, vol. 1176, no. 5, 2019.

[9] M. V. S. Kumar, G. Sanjeev, K. Amarnath, and S. V. Krishna, "Performance Evaluation of an FMS with Alternative Machines using Flexsim Simulation Software," International Journal of Engineering and Management Research (IJEMR), vol. 6, no. 4, 2016.

[10] B. S. Kumar, G. J. Raju, and G. R. Janardhana, "Simulation Modelling and Analysis of Flexible Manufacturing Systems with flexsim software," Research Journal of Engineering and Technology, vol. 9, no. $1,2018$.

[11] S. Huihui, M. Xiaoxia, and M. Xiangguo, "Simulation and Optimization of Warehouse Operation Based on Flexsim," Journal of Applied Science and Engineering Innovation, vol. 3, no. 4, 2016.

[12] H. He and Z. Hu, "Analysis of Fast Food Service Capability Based on Flexsim Modeling and Simulation," IOP Conference Series: Materials Science and Engineering, vol. 394, no. 5, 2018.

[13] L.-H. Chen, D.-W. Hu, and T. Xu, "Highway Freight Terminal Facilities Allocation based on Flexsim," Procedia - Social and Behavioral Sciences, vol. 96, 2013.

[14] F. Zhang and C. Tian, "Study on Modeling and Simulation of Logistics Sorting System Based on Flexsim," International Journal of Advanced Network, Monitoring and Controls, vol. 2, no. 4, 2018. 
[15] G. Yong, Z. Taoxing, W. Dongmei, R. Jianxin, and W. Hui, "Optimization and Simulation of Express Sorting System in Ecommerce Background," Journal of Shijiazhuang Tiedao University(Social ence Edition), 2019.

[16] C. Yanru, X. U. Zhigang, D. Shuhao, Q. Kaizhong, and Y. Deyu, "Optimization of reducer assembly process based on value stream mapping technology," Manufacturing Technology \& Machine Tool, 2019.

[17] G. E. Xiao-Mei, L. I. Qian-Long, Z. Xi, H. Jia-Li, and Z. Zhe, "Optimization and Simulation of Movable Cabinet Assembly Line Based on Flexsim," Value Engineering, 2019.

[18] H. Wei-Jian and X. U. Jian-Zhou, "Research on Unattended Warehouse Simulation Based on Flexsim," Logs Engineering and Management, 2019.

[19] T. U. Tian-Hui, X. U. Ze, H. Lei, F. U. Jian-Lin, and D. Guo-Fu, "The Simulation and Optimization of Wheelset Pressing Production Line Based on Flexsim," Modular Machine Tool \& Automatic Manufacturing Technique, 2019.

[20] O. Riskadayanti, Yuniaristanto, W. Sutopo, and M. Hisjam, "Discreteevent simulation of a production process for increasing the efficiency of a newspaper production," IOP Conference Series: Materials Science and Engineering, 2019.

[21] Z. Lin, D. Pengzheng, H. U. Lingling, S. Liming, and Y. U. Xiaohong, "Analysis and Optimization of Sofa Production Operation Based on Flexsim," Furniture, 2019.
[22] Z. Kangqu, Y. Kun, and Y. Siqi, "Simulation and Optimization of Micro-Cultivator Packing Line of HTBS," Industrial Engineering Journal, 2019.

[23] Q. Jin and T. T. Li, "Operation Improvement of Third-Party Logistics Enterprise Based on Activity-Based Costing Method," in ICMSS 2020: 2020 4th International Conference on Management Engineering, Software Engineering and Service Sciences, 2020.

[24] W. Geng, X. U. Cai-Guang, C. F. E. Co, and Ltd, "Simulation study on the capacity of Tonggu channel in Shenzhen Port based on Flexsim," Port \& Waterway Engineering, 2019.

[25] B. Bing-Liang and L. Lin, "Optimization of Motor Assembly Line Balance Based on Flexsim Simulation," Value Engineering, 2019.

[26] J. Liu, G. Lu, and H. Wang, "Simulation of Automated Stereo Warehouse System Based on Flexsim," 2018.

[27] J. Cong and C. Er-Wei, "Flexsim Simulation-based Assembly Shop Process Optimization," Logs Engineering and Management, 2018.

[28] Y. Yi, L. Dingyi, and S. University, "Research on Sorting Flow Simulation of Campus Common Distribution Service Station Based on FlexSim," Logistics Sci-Tech, 2018.

[29] Z. Ruihan and T. Xiaowei, "Application of Flexsim simulation in Shanghai Pudong International Airport ground taxiing optimization," Journal of Civil Aviation University of China, 2018.

[30] F. Dianjun, L. Wei, and Z. Xinyan, "Simulation and Optimization of Plant Logistics and Transportation Processes Based on Flexsim," Logs Technology, 2018. 